\title{
Using fMRI to characterize how cortex represents limb motions
}

\author{
Samir Menon ${ }^{1 *}$, Jack Zhu' ${ }^{1}$ Paul Quigley ${ }^{1}$, Franco Pestilli', Kwabena Boahen ${ }^{3}$, Oussama Khatib ${ }^{1}$ \\ From The Twenty Third Annual Computational Neuroscience Meeting: CNS*2014 \\ Québec City, Canada. 26-31 July 2014
}

Neuroimaging experiments that map limb motions on to the brain observe fractured somatotopic maps, with correlated neural responses across functionally related joints in the arm [1]. Analyzing such experiments involves visually comparing winner-takes-all neural activation maps for dif- ferent subjects that are generated with generalized linear models [2]. Such analyses, however, abstract cross-joint correlations and treat reliable deviations from canonical neural (haemodynamic) response functions as temporal noise. Here, using classification accuracy while delineating
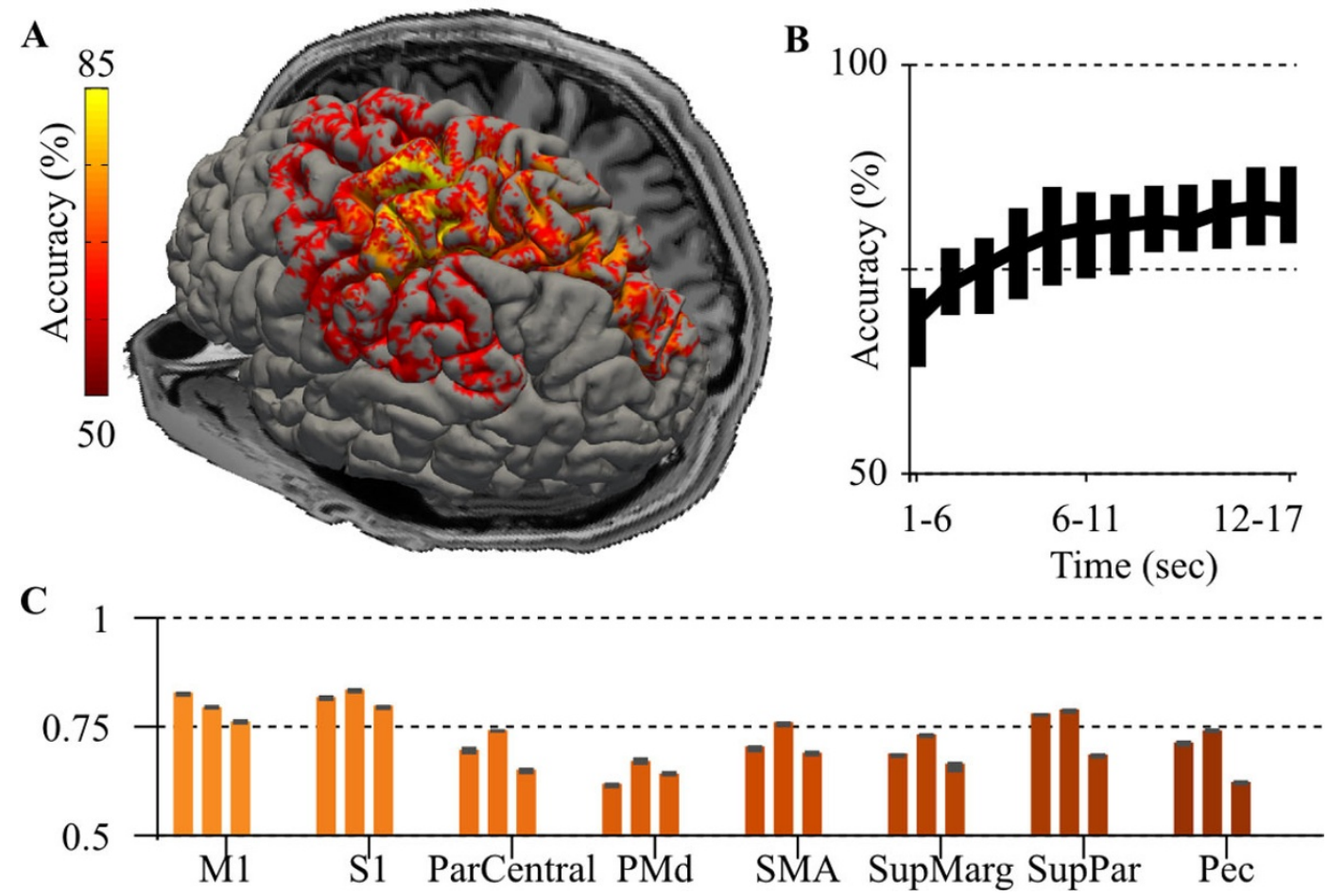

Figure 1 Motor classification. A. Classification accuracy by region for a subject. B. Later stage fMRU neural responses improve classification accuracy. A sliding window of time-series data used increases accuracy till 15 seconds. C. Medican and 95\%ile accuracies are shown for three subjects across a variety of regions.

\footnotetext{
* Correspondence: smenon@stanford.edu

${ }^{1}$ Department of Computer Science, Stanford University, Stanford, CA, 94305,

USA

Full list of author information is available at the end of the article
} 
different limb motions as a metric, we demonstrate that the peak neural response amplitude-upon which winnertakes-all analyses are based-is the least informative part of the time-series. In contrast, our experiments suggest that neural responses are most informative after the initial response peak ( $\mathrm{t}=4-10 \mathrm{~s})$. Our observations extend to primary motor (M1), pre-motor (PMd), somatosensory (S1), superior parietal (SupPar), and supplementary motor (SMA) cortices, matching prior region-agnostic results [3]. As expected for open-loop limb motions, median M1 and S1 classification accuracies are greater than SupPar, PMd and SMA. All accuracies exceed the ventricles, which set a data-driven noise threshold at chance (50-55\% accuracy; chance $=50 \%$ ) and demonstrate that our datasets lack taskcorrelated noise.

Our results suggest that Functional Magnetic Resonance Imaging (fMRI) time-series responses convey sufficient information to classify a variety of motor tasks in regions where neural activity is expected to be correlated across conditions. Reproducing our results, however, may require fMRI datasets with minimal $(<1 \mathrm{~mm})$ head-motion, no spatial smoothing, and tests for null (baseline) results in regions with no expected effect.

\section{Methods}

We used fMRI to scan three subjects who moved their wrist, elbow or shoulder up and down, or rotated their wrist or shoulder while holding two different weights (50g, 500g. 8s*32trials*10conditions). We used Freesurfer's Desikan-Killiany atlas to identify motor regions and iterated over each region in $1 \mathrm{~cm}^{3}$ sections, using randomly sampled voxels (40 bootstraps; 50 of $\sim 150$ voxels) and a maximum-margin hyperplane with leave-one-out crossvalidation to pair-wise classify limb motions. We also compared random (unbiased) responses with reliable voxels selected using a traditional general linear impulse response model.

\section{Authors' details}

${ }^{1}$ Department of Computer Science, Stanford University, Stanford, CA, 94305, USA. 'Department of Psychology, Stanford University, Stanford, CA, 94305, USA. ${ }^{3}$ Department of Bioengineering, Stanford University, Stanford, CA, 94305, USA.

Published: 21 July 2014

\section{References}

1. Meier JD, Graziano MS: A. e. Complex organization of human primary motor cortex: A high-resolution fMRI study. J Neurophysiology 2008, 100:1800-1812.

2. Strother L, Medendorp WP, Coros AM, Vilis T: Double representation of the wrist and elbow in human motor cortex. Eur J Nsci 2012, 36(9):3291-3298.

3. Brantner G, Menon S, Schorpp G, Khatib O: SVM-based classification of motor-tasks on fmri-bold data. IEEE EMBS Neural Engineering 2013.

doi:10.1186/1471-2202-15-S1-P126

Cite this article as: Menon et al:: Using fMRI to characterize how cortex represents limb motions. BMC Neuroscience 2014 15(Suppl 1):P126.

\section{Submit your next manuscript to BioMed Central} and take full advantage of:

- Convenient online submission

- Thorough peer review

- No space constraints or color figure charges

- Immediate publication on acceptance

- Inclusion in PubMed, CAS, Scopus and Google Scholar

- Research which is freely available for redistribution 